OPEN

SUBJECT AREAS:

SYSTEMS BIOLOGY

NEUROSCIENCE

PLASTICITY

MEDICAL RESEARCH

Received

2 December 2011

Accepted

30 January 2012

Published

23 February 2012

Correspondence and requests for materials should be addressed to D.L. (dlevi@berkeley. edu)

\section{Training the brain to overcome the effect of aging on the human eye}

\author{
Uri Polat ${ }^{2}$, Clifton Schor ${ }^{1}$, Jian-Liang Tong' ${ }^{1}$, Ativ Zomet ${ }^{1,2}$, Maria Lev $^{2}$, Oren Yehezkel ${ }^{2}$, Anna Sterkin ${ }^{2}$ \\ \& Dennis M. Levi ${ }^{1}$ \\ ${ }^{1}$ School of Optometry and Helen Wills Neuroscience Institute, UC Berkeley, Berkeley, CA, ${ }^{2}$ Faculty of Medicine, Goldshleger Eye \\ Research Inst, Tel-Aviv University, Tel-Hashomer, Israel.
}

Presbyopia, from the Greek for aging eye, is, like death and taxes, inevitable. Presbyopia causes near vision to degrade with age, affecting virtually everyone over the age of 50. Presbyopia has multiple negative effects on the quality of vision and the quality of life, due to limitations on daily activities - in particular, reading. In addition presbyopia results in reduced near visual acuity, reduced contrast sensitivity, and slower processing speed. Currently available solutions, such as optical corrections, are not ideal for all daily activities. Here we show that perceptual learning (repeated practice on a demanding visual task) results in improved visual performance in presbyopes, enabling them to overcome and/or delay some of the disabilities imposed by the aging eye. This improvement was achieved without changing the optical characteristics of the eye. The results suggest that the aging brain retains enough plasticity to overcome the natural biological deterioration with age.

$\mathrm{P}$ resbyopia results from the gradual decrease of accommodative (focusing) power with age ${ }^{1}$. This loss of accommodation begins quite early in life, and is complete or nearly so by about age 50 , with the firstreported negative effects on reading and near tasks occurring between about 42 and 44 years of age ${ }^{2}$, and affecting virtually everyone by age 51 . It is estimated that by 20201.4 billion people will be affected by presbyopia ${ }^{3}$. The age-related reduction in accommodation results in reduced near visual acuity and reduced reading abilities with no optical correction. Indeed, the most common symptom of presbyopia is difficulty in reading up close, particularly in poor lighting, and early presbyopes often complain that their arms are too short. The most common solution is reading glasses or bifocals.

In presbyopia, the visual input to the cortex is limited by the optics of the eye. High spatial frequencies (fine details) are attenuated. Thus, at near, the visual acuity and contrast sensitivity of uncorrected presbyopes is lower than normal ${ }^{4}$ (see also Figs. 1 and 2). Since contrast is important in driving neural responses in the visual cortex, the consequence of a blurred input may result in weaker and slower neuronal responses in the visual cortex, leading to degraded letter identification and reduced reading abilities ${ }^{4}$.

We reasoned that if the neural signals from the blurred retinal image could be boosted or used more efficiently by the brain, it may be possible to overcome or at least delay the effects of presbyopia. To test this idea, we employed a training protocol (perceptual learning) on 30 presbyopes (age $51 \pm 4.4$, mean \pm se; see Methods). Perceptual learning with similar, though not identical, tasks has been shown to improve visual functions in normal vision and in patients with both neural (amblyopia) ${ }^{4,5}$ and optical (low myopia and presbyopia) ${ }^{4,6}$ deficits.

Based on the previous studies ${ }^{4}$, participants were asked to practice at least 3 times per week, on different days, for about 30 minutes per session. The training was based on detection of a small low-contrast grating patch $(\mathrm{T})$ flanked by two similar high-contrast patches $(\mathrm{M})$ positioned in a collinear configuration that is known to result in facilitation of target detection (LM - see Supplementary Fig. 1) ) $^{7-9}$. In addition, we imposed a condition of backward masking with different inter-stimulus intervals, in which the flankers were delayed after the target (BM-T) and a condition when a second pair of mask followed the LM configuration (BM-LM) ${ }^{10,11}$. The BM effect was shown earlier to cancel the facilitation effect after a delay (ISI) of $50 \mathrm{~ms}^{12}$. Thus, the purpose of the training was also to try to induce faster processing to overcome the masking effect. A similar training protocol resulted in robust gains in perceptual functions, such as percent correct, sensitivity and faster reaction time in young participants $^{13}$. Thirty subjects completed $37.4 \pm 10.7$ (mean \pm std) sessions over a 3 months period. In order to assess the effects of the training, we pre- and post-tested our subjects on a number of behavioral tests (visual acuity, reading speed, contrast detection and contrast discrimination) as well as on tests of accommodation, pupil size and depth of focus (see Methods). 


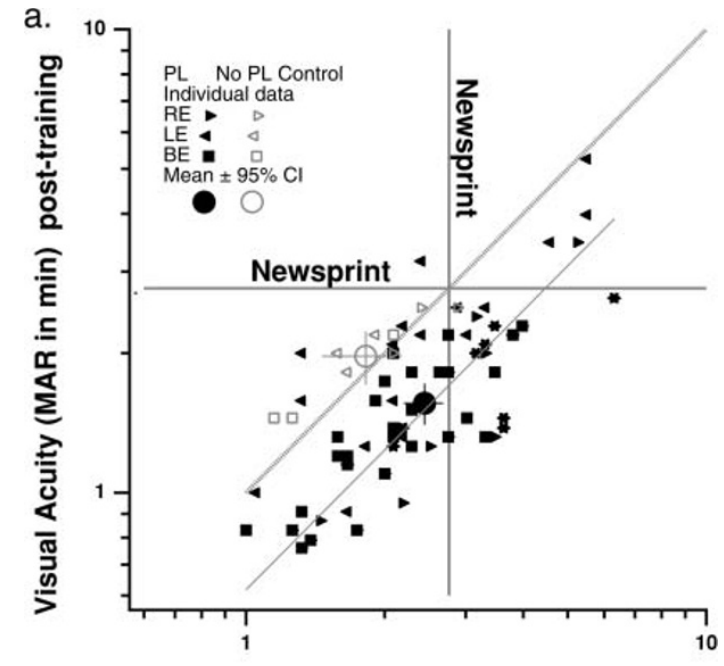

b. Visual Acuity (MAR in min) pre-training

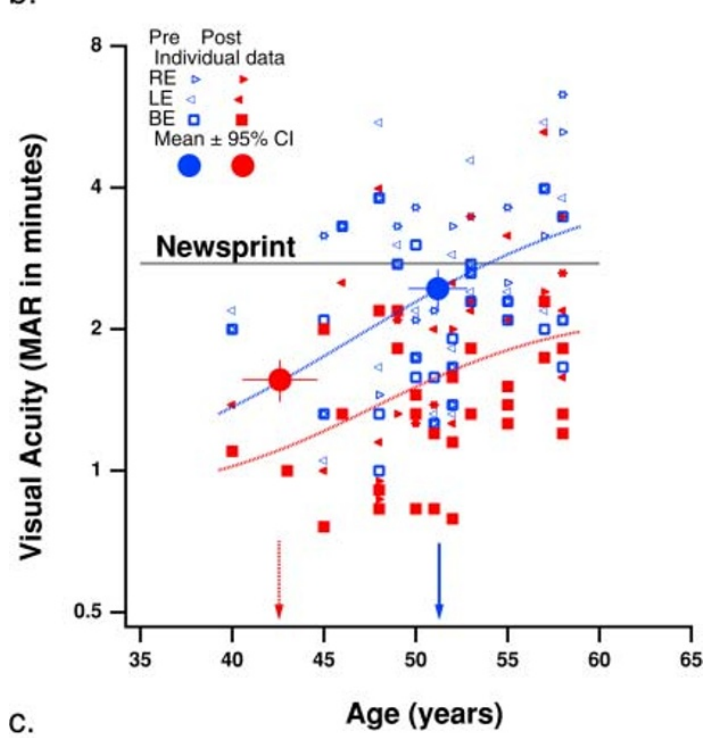

c.

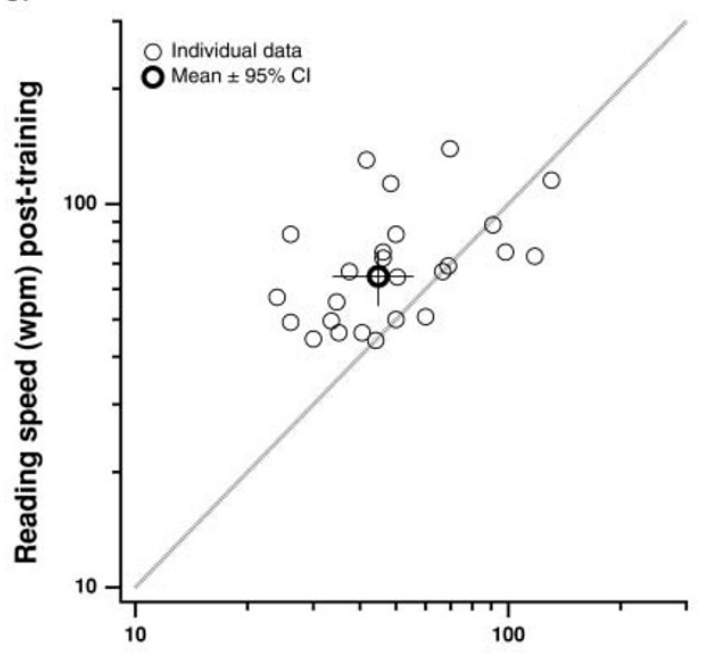

Reading speed (wpm) pre-training

\section{Results}

Near Visual Acuity and reading. The major difficulty in presbyopia is reading small print. Consider reading the newspaper. The font size in a typical newspaper has a visual angle of $\approx 14$ minutes (and a stroke size of $\approx 2.8$ minutes) when viewed from a distance of

Figure $1 \mid$ (a) Near visual acuity before (abscissa) and after (ordinate) perceptual learning (PL). Solid symbols are presbyopic subjects (median age 51). Open symbols are the no PL control group. The dotted gray line is the quality line. The solid gray diagonal is a power function fit to the presbyopes data. The horizontal and vertical lines show typical newsprint size (expressed in minutes of arc). (b) Near visual acuity vs. age before (blue) and after (red) PL. The large blue and red circles show the geometric mean acuities before and after PL, plotted at the corresponding pretraining abscissa values (shown by the arrows). (c) Reading speed before (abscissa) and after (ordinate) PL for the smallest letter size that each subject could read.

$40 \mathrm{~cm}^{18}$. This was too small for glasses-free reading in many of our subjects prior to training. Fig. 1a shows near visual acuity (expressed as the minimum angle of resolution) before (abscissa) and after (ordinate) training. Newspaper font size (expressed as the stroke size, for comparison with acuity) is shown by the gray lines. Note that for a number of the participants, the newsprint size was smaller than letter sizes for their pre-training acuity (indicated by the data to the right of the vertical gray line). The mean visual acuity before training (large solid circle) is just below the newsprint size. Importantly, for every participant, the post-training acuity letter size was better (smaller) than the pre-training acuity letter size, on average by a factor of $\approx 1.6$, from $2.44 \pm 0.24$ (geometric mean \pm $95 \%$ confidence interval) arc min at pre-test to $1.56 \pm 0.16 \mathrm{arc}$ min at post-test ( $\mathrm{p}<1.6 \mathrm{e}-19$ ) (Fig. 1a). Thus, after training, newsprint was substantially larger than acuity letter size (indicated by the data below the horizontal gray line) enabling glasses-free reading with both eyes (squares) for all subjects.

In order to ensure that the improvements were due to the training, rather than simply due to variability in the pre- and post- test measurements, we pre- and post-tested the visual acuity and contrast sensitivity of three observers approximately two months apart, but with no intervening training. The visual acuity data of these "control" subjects are shown by the gray symbols in Fig. 1a. Note that their data fall along the equality line, and the pre and post-training measures were not significantly different ( $p$ $=0.16)$.

Presbyopia is an age related visual impairment, so it is instructive to look at how uncorrected near visual acuity depends on age (Fig. 1b). This figure shows clearly that both before (blue) and after (red) training, uncorrected near acuity deteriorates systematically with age. Importantly, at every age, acuity is better (lower) after training, and newsprint is not too small for any subject using both eyes (red squares). We fit the visual acuity (VA) vs. age data with a function of the form:

$$
\mathrm{VA}=\mathrm{VA}_{\mathrm{b}}+\left(\mathrm{VA}_{\max } / 1+\exp \left(\left(\text { Age }_{50}-\text { Age }\right) / \mathrm{R}\right)\right)
$$

where $\mathrm{VA}_{\mathrm{b}}$ and $\mathrm{VA}_{\text {max }}$ are the baseline and maximum visual acuities respectively; Age $_{50}$ is the age at which visual acuity reaches half of its maximum value, and $\mathrm{R}$ is the rate of decline with age. The fits are shown by the dotted red and blue curves in Fig 1b. The downward shift in acuity at each age following perceptual learning (PL) can be clearly seen in the fits. Interestingly, the mean VA was reduced from $2.44 \pm 0.24$ to $1.56 \pm 0.16$, equivalent to an effective reduction in age of $\approx 8.6$ years, as indicated by the horizontal positions of the mean acuities (large solid circles) and arrows in Fig. 1b.

Since visual processing time is slower in presbyopia ${ }^{4}$, we wondered whether reading speed would be improved by our training. In order to test this, we measured reading speed using the MNREAD chart ${ }^{19}$ and found that the reading speed of presbyopes with uncorrected near vision was slow prior to training when measured on the smallest letter size that subjects could read, but it improved, on average by about 17 words/minute following training (Fig. $1 \mathrm{c}-\mathrm{p}=0.0035$, one-tailed t-test). After training, 
a.

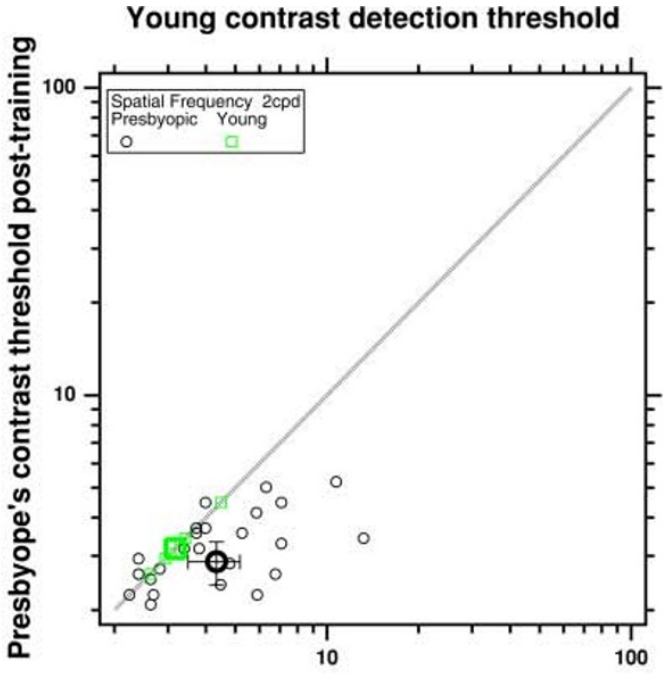

Presbyope's contrast threshold pre-training Young contrast detection threshold

b.

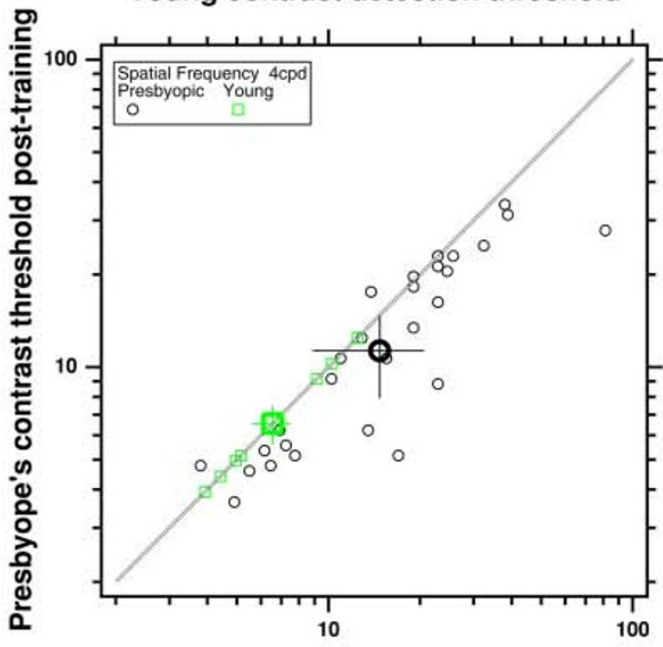

Presbyope's contrast threshold pre-training

C.

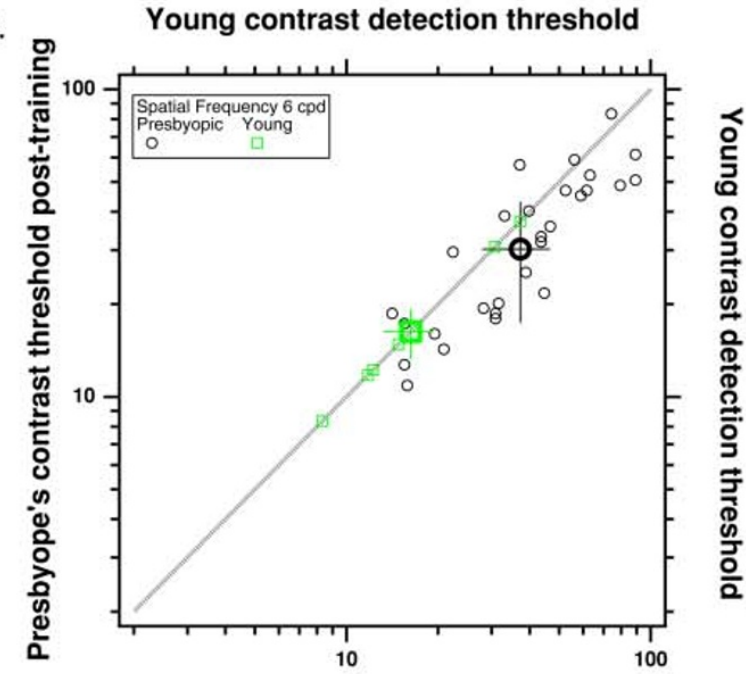

Presbyope's contrast threshold pre-training d.

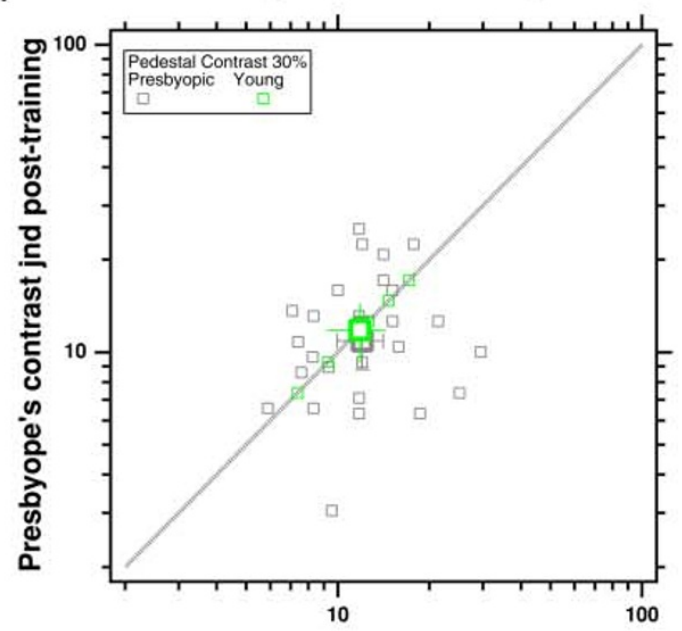

Presbyope's contrast jnd pre-training

Young control contrast jnd

e.

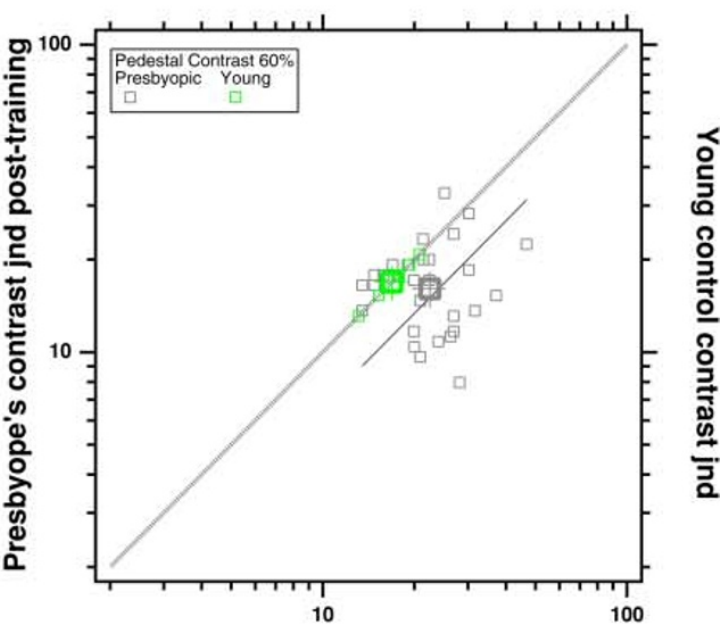

Presbyope's contrast jnd pre-training

f.

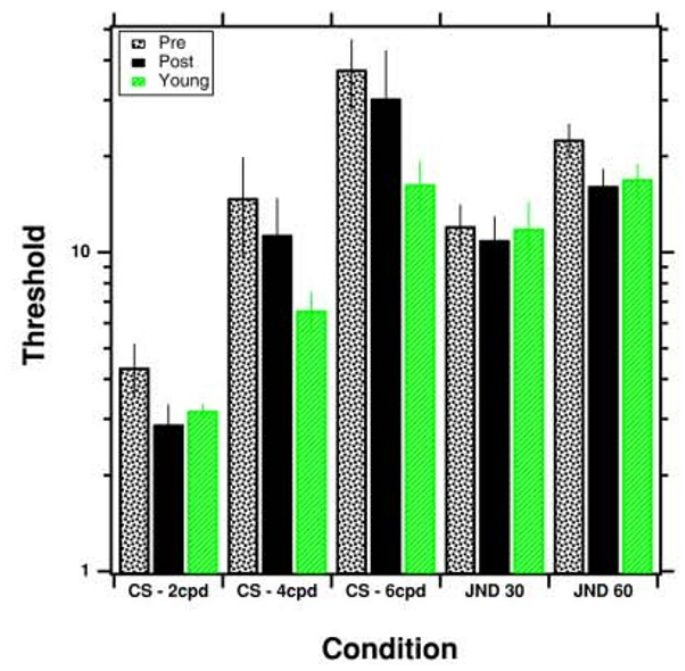

Figure $2 \mid$ Presbyope's contrast thresholds (black symbols) before (bottom abscissa) and after (left ordinate) PL for spatial frequencies of 2 (a), 4 (b) and 6 (c) cpd. For comparison thresholds of the young control subjects (green symbols) are plotted along the top abscissa and right ordinate, so they fall along the equality line (gray dotted line). d, e. Contrast discrimination thresholds (jnds) (gray symbols) before (bottom abscissa) and after (left ordinate) PL for a spatial frequencies of $4 \mathrm{cpd}$ and pedestal contrasts of $30 \%$ (d) and $60 \%$ (e). Thresholds of the young control subjects (green symbols) are plotted along the top abscissa and right ordinate, so they fall along the equality line (gray dotted line). f. Summarizes the thresholds for each condition. 

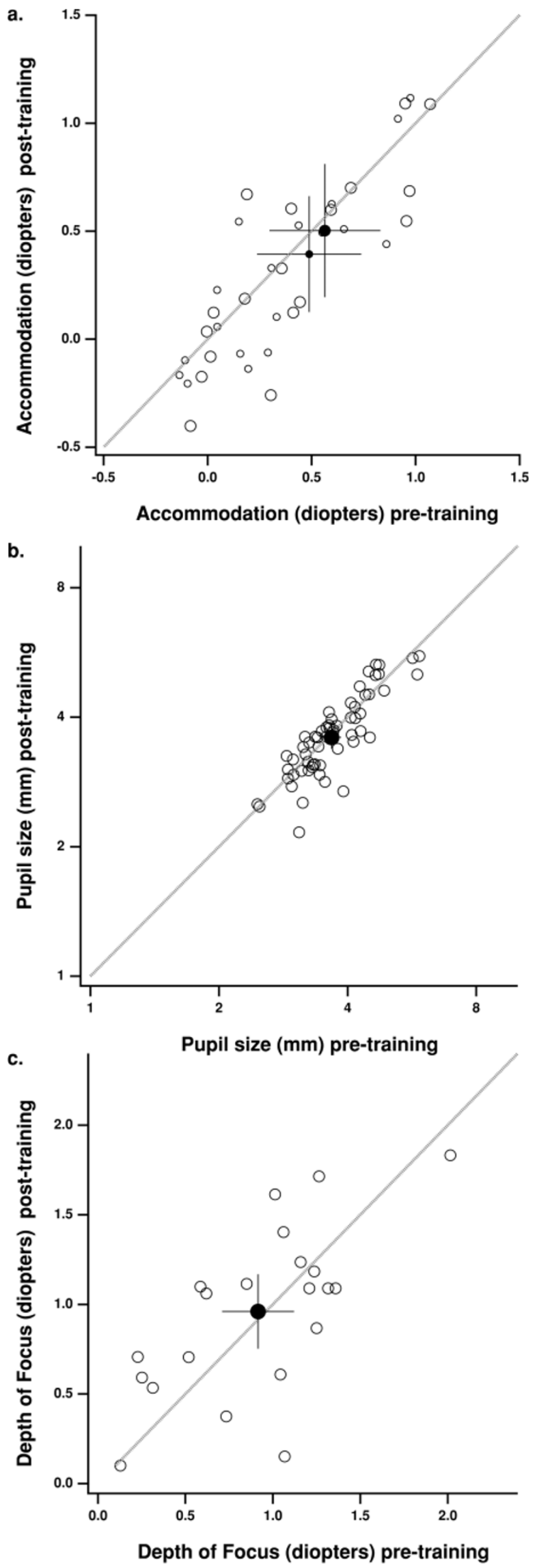

Figure $3 \mid$ Accommodation (a), pupil size (b) and depth of focus (c) before (abscissa) and after (ordinate) perceptual learning (PL). a presbyope would save about 9 minutes when reading a 2000 word article at the smallest letter size!

Contrast detection. After training, contrast detection thresholds improved (decreased) at all 3 tested spatial frequencies $(\mathrm{p}<0.002$, 0.009 and 0.001 for 2,4 and $6 \mathrm{cpd}$, respectively - 1-tailed t-test Fig. $2 \mathrm{a}-\mathrm{c}$ ). This is an improvement of $\approx 34,23$ and 19 percent at spatial frequencies of 2, 4 and $6 \mathrm{cpd}$, respectively. Before treatment, the presbyopes contrast thresholds were significantly higher than that of the young subjects $(\mathrm{N}=7$, age $23.4 \pm 1.4$ years, mean \pm se, shown in green in Fig. 2) for the two higher spatial frequencies ( $\mathrm{p}=$ $0.07,0.01$ and 0.02 for 2,4 and $6 \mathrm{cpd}$, respectively, two-sample twotailed t-test). Following treatment this difference was reduced at the two higher spatial frequencies ( $p=0.05$ and 0.05 for 4 and $6 \mathrm{cpd}$ respectively; two-sample two-tailed t-test), and the presbyopes thresholds were not significantly different from the young subjects at the lowest spatial frequency $(2 \mathrm{cpd} ; \mathrm{p}=0.15)$. These results are consistent with earlier reports of improvement of contrast sensitivity in presbyopic ${ }^{4}$ and amblyopic ${ }^{4,14}$ participants after training. In contrast, our three "no practice control" subjects actually showed a slight (on average $\approx 15 \% \pm 8 \%$ ) worsening of contrast detection thresholds.

Contrast discrimination. The ability to discriminate between grey levels (contrast just noticeable difference or JND) is very important in our daily activities. Whereas previous reports on training on contrast discrimination resulted in inconsistent results ${ }^{15-17}$, here we show, for the first time, improvement in suprathreshold contrast discrimination without direct training on a just-noticabledifference (JND) task (by $28.5 \%$ at the highest pedestal contrast of $60 \%$, fig. 2e). JND remained unchanged for the pedestal contrast of $30 \%(\mathrm{p}=0.526)$, however this threshold was not significantly different from the level of the young controls either for pre-test ( $p$ $=0.636)$ or post-test $(\mathrm{p}=0.933)$ (Fig. 1d). Furthermore, whereas the contrast detection threshold at post-test remained significantly different from the level of young controls $(\mathrm{p}=0.001$ and 0.003 , for pre-test and post-test, respectively - Fig. 2b), the contrast discrimination threshold for the pedestal of 60\%, which was significantly higher than the level of young controls at pre-test ( $p$ $=0.001$ ), was similar to the level of controls following the treatment $(\mathrm{p}=0.952)$. Fig. $2 \mathrm{f}$ summarizes the pre- and post-test thresholds of the presbyopic subjects, along with thresholds of the young controls.

Accommodation, Pupil size and Depth of Focus. Since presbyopia is an optical problem, an intriguing question is whether the improvement of near visual functions following the training resulted from changes in the optical functions of the eye. In order to address this possibility, we tested accommodative power, pupil size and depth of focus, which are known to affect perception. As shown in Fig. 3, there were no changes in our objective measures of accommodation ( $\mathrm{p}=0.409,0.623$ and 0.960 for the distances of 300 , 40 and $33 \mathrm{~cm}$, respectively - Fig. $3 \mathrm{a})$, pupil size ( $\mathrm{p}=0.146,0.872$ and 0.995 for the distances of 300, 40 and $33 \mathrm{~cm}$, respectively - Fig. 3b) or depth of focus ( $p=0.605$; Fig. $3 c$ ). Therefore we conclude that perceptual changes occur due to changes in the brain.

\section{Discussion}

Our results, consistent with previous studies, show that perceptual learning can improve visual acuity and contrast sensitivity in persons with presbyopia, and in some cases, result in performance levels similar to the young control group. Moreover, here we show that training also improves suprathreshold contrast discrimination and reading speed for small letters. Our study is the first to show conclusively that these improvements are not due to improved optical performance of the eye (accommodation, pupil size or depth of focus). 
The uncorrected presbyopic neural system operates under nonoptimal conditions and is limited by the blurred input with lower contrast and resolution, as indicated by the reduced contrast detection and discrimination thresholds, reduced visual acuity and slower reading speed. The effects of training show that the visual system has the potential to operate on the blurred input to reestablish the normal "pre-presbyopic" level, matching the processing of the young group and achieving normal or near normal visual performance for low spatial frequencies and suprathreshold contrasts. Our results underscore the remarkable capacity of the brain to increase the efficiency of neural processing in order to perform "de-blurring" of highly blurred images, retrieve the information and deliver it downstream for further processing at sensory and cognitive levels.

How can we account for the improvement with training? One possibility is that the perceptual learning triggers or enhances the process of blur adaptation. It is now well established that the visual system can adapt to blur, including the blur imposed by it's own optics $^{20-22}$. However we think this is unlikely to account for the improvement because blur adaptation is transient, short-lasting, and if adaptation to presbyopic blur did occur, presbyopes would not have difficulty reading. Rather, we suggest that the improvement is an effect of "de-blurring". This could be achieved by increasing the sensitivity or gain of neurons by a factor that enables image processing at very low signal-to-noise levels with efficiency similar to processing at normal signal-to-noise levels. Support for this possibility is found in the improved contrast discrimination and sensitivity of presbyopes so that following training they are close to the level of the young group. Watson \& Ahumada ${ }^{24}$ conclude that blur detection and discrimination are instances of contrast detection, so it is not unreasonable to assume that when we improve contrast detection through perceptual learning, we also improve the ability to detect and discriminate blurred images.

Increasing sensitivity may, in turn, increase neural processing speed $^{2}$. It is also possible that the training regimen may increase processing speed directly. It was shown earlier that training improved contrast sensitivity ${ }^{4,14,23}$ and, recently, that training on backward masking decreased the latency by $20 \mathrm{~ms}$ in the human brain ${ }^{13}$ and shortened the reaction time ${ }^{4}$. Thus, altogether, training may improve both the sensitivity and processing speed of the presbyopic visual system in order to compensate for the optically degraded visual input transmitted by the aging eye. We note that our study has limitations since subjects were not randomly assigned into experimental and control groups, and our "no practice control" group was small. Thus, a large randomized clinical trial may be useful to confirm the benefit of this approach to a clinical population. Nonetheless, our study provides evidence that while a loss of accommodation is an inevitable consequence of aging, it may be possible to overcome and/or delay the unwanted effects of presbyopia and improve the quality of life in the aging population.

\section{Methods}

We have developed a structured perceptual learning treatment method for improving visual functions in presbyopia ${ }^{4,12}$. Subjects were trained on contrast detection of Gabor targets under backward masking conditions, posing temporal constraints on the visual processing. The training covered a range of spatial frequencies and orientations that were modified in accordance with the performance improvement. Subjects were trained in a dark room from a distance of $40 \mathrm{~cm}$ with both eyes open. Visual acuity, spatial and temporal contrast sensitivity, contrast discrimination and reading speed were tested before (pre-test) and after (post-test) the treatment. Accommodation, depth of focus, and pupil size were also measured objectively at pretest and post-test. Subjects practiced for at least two sessions of about $30 \mathrm{~min}$ per week. On average, subjects practiced for $37.4 \pm 10.7$ (mean \pm std) sessions over 3 months. The study was performed at the University of California, Berkeley, and the experiments were approved by the UC Berkeley Committee for the Protection of Human Subjects.

Subjects. Thirty presbyopic subjects ( 14 females and 16 males, age $51 \pm 4.4$ years old, mean \pm se) with no neurological conditions volunteered to participate in the study. Three presbyopic subjects served as controls, participating in pre-and post-testing roughly 2 months apart, but with no intervening training. Seven young subjects (age
$23.4 \pm 1.4$ years, mean \pm se) with normal or corrected-to-normal vision in both eyes served as a young control group. All participants signed the informed consent form which was approved by the UC Berkeley IRB.

Apparatus. For psychophysical measurements, stimuli were presented on a Sony multiscan G400 color monitor $(1024 \times 768$ pixels at a $75 \mathrm{~Hz}$ refresh rate; gamma correction applied) controlled by a PC. The effective size of the monitor was $17^{\prime \prime}$ $(26 \times 35 \mathrm{~cm})$, which, at a viewing distance of $40 \mathrm{~cm}$, subtended a visual angle of $9.9 \times 13.1$ degrees. A Grand Seiko Auto Ref/Keratometer WAM-5500 was used for measuring objective refraction, accommodation and pupil size. A COAS Precision Aberrometer was used for measuring ocular aberrations during attempted accommodation that were used to compute the depth of focus (DOF).

Optometric and optical measurements pre- and post- training. A complete eye examination was performed at pre-test and post-test by an optometrist. The exam included objective refraction, subjective refraction, ETDRS acuity from viewing distance of $40 \mathrm{~cm}$.

Optical measurements included objective measurements of accommodation and pupil size, stimulated by viewing distances of $33 \mathrm{~cm}, 40 \mathrm{~cm}$ and 3 meters. Objective DOF was quantified from defocus curves that were derived from image quality metrics analysis (VSOTF) of the wavefront measured in the steady-state responses in the zero and non-zero accommodation stimulus conditions (viewing distances of 3 meters and $33 \mathrm{~cm})^{25}$. Defocus curves were calculated from the simulated changes in the defocus term of the Zernike polynomial ranging from -3.00 to $+3.00 \mathrm{D}$ in increments of $0.25 \mathrm{D}^{3}$. Each calculation used the pupil size recorded by the aberrometer as measured during the trial. The log-metric was plotted against linear defocus values. The depth of focus was estimated from the defocus range corresponding to a $50 \%$ reduction from the peak of the metric performance.

Psychophysical measurements pre- and post- training: stimuli and paradigms. The stimuli were localized vertically oriented gray-level gratings (Gabor patches, GPs) with equal distribution (STD, $\sigma$, allowing a minimum 2 cycles in the GP), modulated from a background luminance of $40 \mathrm{~cd} / \mathrm{m}^{2}$ (Supplementary Figs. 1a). Psychophysical measurements included: 1 ) a contrast sensitivity task with brief stimuli (presented for $60 \mathrm{msec}$ ) with a spatial frequency of 2, 4 and 6 cycles per degree (cpd, wavelength, $\lambda$ ), using a $2 \mathrm{AFC}$ detection paradigm (Supplementary Figs. $1 \mathrm{~b}$ ) and 2) a contrast discrimination or a just noticeable difference (JND) task for brief stimuli (presented for $60 \mathrm{msec}$ ) with a spatial frequency of $4 \mathrm{cpd}$, using a 2AFC discrimination paradigm with 2 pedestal contrasts: 30 and $60 \%$ (Supplementary Figs. 1c). Separate blocks were used for each spatial frequency and pedestal contrast Target detection contrast threshold was determined for each condition, in a separate staircase for each block. Subjects started each trial by pressing a key on the keyboard. A fixation circle (subtending a visual angle of 0.7 degrees) was presented in the center of the screen for $200 \mathrm{msec}$ and was followed by two intervals with a $500 \mathrm{msec}$ gap between them. Each interval was preceded by a $300 \mathrm{msec}$ blank period, during which background was presented and an additional blank period with temporal jitter of $500 \mathrm{msec}$ on average. A target GP was presented in one of the two intervals, and subjects were asked to report which interval contained the target by pressing a mouse button (left for the first interval and right for second). Across trials, target presentation was equally distributed between the two intervals. Participants were instructed to maintain their fixation in the center of the monitor and avoid eye movements during the trials.

Reading performance was assessed using MNREAD acuity chart (Minnesota Laboratory for Low-Vision Research, University of Minnesota).

Training paradigm. Subjects completed $37.4 \pm 10.7$ (mean \pm std) training sessions on different days (not including the days of pre-test and post-test). Each session included 4 conditions: 1 ) a foveal target presented alone (T) (Supplementary Figs. 1d), 2) lateral masking (LM), composed of T in the presence of two flanking collinear GPs at a contrast of $40 \%$ (Supplementary Figs. 1d), 3) backward masking on target (BM$\mathrm{T}$ ), composed of $\mathrm{T}$ followed by a mask, identical to the two flanking collinear GPs used in LM, presented at varied time intervals (ISIs) after T (Supplementary Figs. 2a) and 4) backward masking on lateral masking (BM-LM), composed of LM followed by another mask, identical to the two flanking collinear GPs used in LM, presented at varied time intervals (ISIs) after LM (Supplementary Figs. 2b). The ISIs were: 60, 90, $120,150,180,210$ or $240 \mathrm{msec}$. A two alternative forced choice (2AFC) paradigm was used, identical to the one used in pre-test and post-test and subjects were asked to report which interval contained the target. Auditory and visual feedback was provided. ISI, duration of target presentation, and masking GPs, their orientation, spatial frequency and spatial separation between them were modified between sessions, one parameter at a time, according to performance in the preceding session. The duration of stimuli presentation varied between 60 to $120 \mathrm{msec}$. The spatial distance between the target and the flankers varied between 2 to $4 \lambda$. The orientation of GPs was always the same for target and masking GPs (i.e., collinear).

Data analysis. The results were first entered into ANOVA; pairwise comparisons were performed using paired one-tailed t-tests, unless specified differently.

Supplementary Results. A two-way ANOVA was performed for the CS task (test (2: pre-test and post-test) $\times$ spatial frequency $(3: 2,4$ and $6 \mathrm{cpd}))$ and for the CD task (test $(2$ : pre-test and post-test) $\times$ pedestal contrast $(2: 30$ and $60 \%))$. For the CS task, there was a significant main effect of the test $(F(1,162)=12.87, p=0.0004)$ and of the 
spatial frequency $(\mathrm{F}(2,162)=5.78, \mathrm{p}=0.004)$. For the CD task, there was also a significant main effect of the test $(F(1,108)=10.41, \mathrm{p}=0.002)$ and of the pedestal contrast $(\mathrm{F}(1,108)=5.93, \mathrm{p}=0.02)$.

1. Koretz, J. F., Kaufman, P. L. Neider, M. W. et al. Accommodation and presbyopia in the human eye - aging of the anterior segment. Vision Res 29, 1685-92 (1989).

2. McDonnell, P. J., Lee, P., Spritzer, K., Lindblad, A. S. \& Hays, R. D. Associations of presbyopia with vision-targeted health-related quality of life. Arch Ophthalmol 121, 1577-1581, doi:10.1001/archopht.121.11.1577121/11/1577 [pii] (2003).

3. Holden, B. A. et al. Global vision impairment due to uncorrected presbyopia. Arch Ophthalmol 126, 1731-1739 (2008).

4. Polat, U. Making perceptual learning practical to improve visual functions. Vision Res 49, 2566-2573 (2009).

5. Levi, D. M. \& Li, R. W. Perceptual learning as a potential treatment for amblyopia: a mini-review. Vision Res 49, 2535-49 (2009).

6. Durrie, D. \& McMinn, P. S. Computer-based primary visual cortex training for treatment of low myopia and early presbyopia. Trans Am Ophthalmol Soc., 105, 132-8 (2007).

7. Polat, U. \& Sagi, D. Lateral interactions between spatial channels: suppression and facilitation revealed by lateral masking experiments. Vision Res 33, 993-999 (1993).

8. Polat, U. \& Sagi, D. The architecture of perceptual spatial interactions. Vision Res 34, 73-78 (1994)

9. Polat, U. \& Sagi, D. Spatial interactions in human vision: from near to far via experience-dependent cascades of connections. Proc Natl Acad Sci U S A 91, 1206-1209 (1994)

10. Polat, U., Sterkin, A. \& Yehezkel, O. Spatio-temporal low-level neural networks account for visual masking. Adv Cogn Psychol 3, 153-165 (2007).

11. Polat, U. \& Sagi, D. Temporal asymmetry of collinear lateral interactions. Vision Res 46, 953-960 (2006).

12. Sterkin, A., Yehezkel, O., Bonneh, Y. S., Norcia, A. \& Polat, U. Backward masking suppresses collinear facilitation in the visual cortex. Vision Res 49, 1784-1794 (2009).

13. Sterkin, A., Yehezkel, O. \& Polat, U. Learning to be fast: gain accuracy with speed. Vision Res in press (2012).

14. Polat, U., Ma-Naim, T., Belkin, M. \& Sagi, D. Improving vision in adult amblyopia by perceptual learning. Proc Natl Acad Sci U S A 101, 6692-6697 (2004).

15. Yu, C., Klein, S. A. \& Levi, D. M. Perceptual learning in contrast discrimination and the (minimal) role of context. J Vis 4, 169-182 (2004).

16. Adini, Y., Wilkonsky, A., Haspel, R., Tsodyks, M. \& Sagi, D. Perceptual learning in contrast discrimination: the effect of contrast uncertainty. J Vis 4, 993-1005 (2004).

17. Adini, Y., Sagi, D. \& Tsodyks, M. Context-enabled learning in the human visual system. Nature 415, 790-793. (2002).

18. Legge, G. E. \& Bigelow, C. A. Does print size matter for reading? A review of findings from vision science and typography. J Vis. Aug 9;11 (5).pii: 8. (2011).
19. Subramanian, A. \& Pardhan, S. The repeatability of MNREAD acuity charts and variability at different test distances. Optom Vis Sci 83, 572-576 (2006).

20. Webster, M. A., Georgeson, M. A. \& Webster, S. M. Neural adjustments to image blur. Nat Neurosci. 5, 839-40 (2002).

21. Elliott, S. L., Hardy, J. L., Webster, M. A., Werner, J. S. Aging and blur adaptation. J Vis. Apr 18;7(6),8 (2007).

22. Mon-Williams, M., Tresilian, J. R., Strang, N. C., Kochhar, P., Wann, J. P. Improving vision: neural compensation for optical defocus. Proc Biol Sci. 265, 717 (1998).

23. Zhou, Y. et al. Perceptual learning improves contrast sensitivity and visual acuity in adults with anisometropic amblyopia. Vision Res 46, 739-750 (2006).

24. Watson, A. B. \& Ahumada, A. J. Blur clarified: a review and synthesis of blur discrimination. J Vis. Sep 19;11(5). pii: 10. doi: 10.1167/11.5.10. (2011)

25. Tahir, H. J., tong, J. L., Geissler, S., Vedamurthuy, I. Schor CM Effects of accommodation training on accommodation and depth of focus in an eye implanted with a crystalens intraocular lens. J Refract. Surg. 26(10), 772-779 (2010)

\section{Acknowledgements}

This research was supported by Ucansi Inc. (UP), and grants from the National Eye Institute, NIH, RO1EY01728 (DL) and RO1EY017678. (CS). We thank Roger Li for his thoughtful comments and suggestions on an earlier version of the manuscript.

\section{Author contributions}

DL, UP and CS were involved in designing the study. DL, UP, AS and CS were involved in the writing and editing. JLT, AZ, ML, OY, AS were involved in collecting and analyzing the data. All authors reviewed the manuscript.

\section{Additional information}

Supplementary information accompanies this paper at http://www.nature.com/ scientificreports

Competing financial interests: UP was funded in part by Ucansi Inc. He received compensation as a part-time employee and owns stock in the company. The other authors declare no competing financial interest.

License: This work is licensed under a Creative Commons

Attribution-NonCommercial-NoDerivs 3.0 Unported License. To view a copy of this license, visit http://creativecommons.org/licenses/by-nc-nd/3.0/

How to cite this article: Polat, U. et al. Training the brain to overcome the effect of aging on the human eye. Sci. Rep. 2, 278; DOI:10.1038/srep00278 (2012). 